\title{
Psychodiagnosis in hypothetical problem spaces
}

\author{
BENJAMIN KLEINMUNTZ \\ Department of Psychology, University of Illinois, Chicago, Illinois 60680
}

and

DON N. KLEINMUNTZ

Center for Decision Research, University of Chicago, Chicago, Illinois 60637

\begin{abstract}
Computer uses are demonstrated for designing a hypothetical psychodiagnostic system that can function in artificially created mental health environments. The idea is to simulate both a psychodiagnostic system and a clinician's strategies in order to learn about computer as well as human information processing. An inductive method for teaching humans the elements of the diagnostic system and its solutions is also introduced.
\end{abstract}

Our prior research in the use of computers in mental health-related areas focused on personality test interpretation by computer (B. Kleinmuntz, 1963, 1969), automated personality test administration (B. Kleinmuntz \& McLean, 1968), and computer interviewing (Brooks \& B. Kleinmuntz, 1974; B. Kleinmuntz, 1975). All of these studies had as their main focus the practical aim of providing a service to the field of mental health. As such, they concentrated on applying the state-of-the-art software and hardware developments to our perceived needs in mental health and delivery.

This paper has no such practical aim. Our purpose here is to conceptualize a hypothetical psychodiagnostic problem space that can be input to a computer along with several diagnostic strategies. In this sense, the hypothetical space will be comparable to the American Psychiatric Association's (1980) third revision of the Diagnostic and Statistical Manual of Psychiatric Disorders (DSM III). The diagnostic strategies are comparable to clinicians examining patients in order to reach a classificatory decision about them.

\section{RATIONALE}

The rationale for using a hypothetical system rather than the actual one is that we can control the parameters of the system in order to change its features along prespecified dimensions. The changes that will be made in the system are designed to increase or decrease the levels of cognitive complexity for human diagnosticians, on the one hand, and to increase or decrease the computer's computational burden, on the other hand. Eventually, we want also to compare the cost-effective tradeoffs between computer and human optimality. That is, we want to be able to ascertain at which level of complexity of a problem space it becomes more or less optimal to use computer vs. human decision making capabilities. A more detailed description than is possible here of the computer and human decision making systems appears elsewhere (D. Kleinmuntz \& B. Kleinmuntz, 1981).

\section{COMPUTER STUDIES OF HYPOTHETICAL PROBLEMS}

The mental health problem space was constructed by assigning symptoms and signs to disorders; the precise numbers of these were determined by the complexity of the environment desired for any particular problem. Each symptom has assigned to it a probability of association to a disease. Configurations of symptoms in various combinations are also given probabilities that change as a function of given base rates. Base rates are adjustable values that may be subject to regional changes or may change depending on what particular mental health installation is being postulated.

Case history data and information obtained from observations, interviews, and tests have varying diagnostic values that can be adjusted according to the complexity of the problem desired. Each datum and test has prescribed predictive validities and reliabilities assigned to it.

Treatments for particular disorders were assigned, and these have diagnostic and therapeutic values attributed to them. If the treatment is somatic rather than psychological, risk factors associated with its administration and withholding were also assigned, depending on the treatment's past and potential harm to patients. Important criteria of a good case history, test, and treatment are that they result in a correct diagnosis and lead to proper treatment. Incorrect diagnoses and improper treatments move the patient further away from good mental health. The latter is defined by a scale ranging from 0 (very good mental health; i.e., no symptoms of 
disorder) to 99 (very poor mental health). Deterioration and improvement in a patient's condition are measurable in terms of quantitative movements toward and away from very good mental health, respectively. Some patients deteriorate if left undiagnosed and untreated; others show spontaneous remission if they are left alone. Precisely which happens under what circumstances is varied by the experimenter, depending on the prespecified parameters of a particular computer problem.

Values were also assigned to improvements in the quality of a patient's life, and these quality-of-life considerations are traded off with the probability of risking death or discomfort in the case of somatic treatment or monetary cost in the case of psychotherapy.

Since we also are interested in clinicians as decision makers, their design is equally critical. The clinicians appear as decision rules of three types: (1) expected utility Bayesian rules, (2) heuristic search strategies, and (3) generate-and-test strategies. Only the last of these will be touched on briefly here; discussion of the other two types of decision strategies would take us too far afield into computational details that are beyond the scope of the present paper (again, see D. Kleinmuntz \& B. Kleinmuntz, 1981).

The generate-and-test treatment strategy makes minimal demands on the decision maker, regardless of whether this is a computer or a human. It requires negligible knowledge and very little in the way of cognitive effort. A treatment is generated at random and is tested as long as the results are favorable. If the observed effectiveness of a treatment falls below a minimal level, a new treatment is selected. This continues, always keeping a treatment as long as it seems to be effective. The symptoms of the patient are ignored, and no attempt is made to diagnose. This is in sharp contrast to the expected utility Bayesian and heuristic strategies, which require extensive computational or human effort to diagnose a disorder before assigning a treatment. This generate-andtest treatment strategy is a very simple example of trial-and-error learning.

\section{TEACHING PROBLEMS AND SOLUTIONS TO HUMANS}

So that we can compare humans with computers in solving hypothetical problems, we must first teach humans the features and the solutions to problems such as those described above. We can do this in one of two ways: by means of the deductive or inductive method. Learning by deduction requires that inferences be made on what the data imply as well as what information the data contain; learning by induction involves reasoning from the particulars of the data base to the general. The latter involves the process of discovery by observing instances and features of a data space. Teaching a computer or a human by induction rather than deduction suits our research purposes quite well, because it permits these problem solvers to extract information from specific examples of the environment in much the same way that problem solvers form connections from characteristics of the task environment to solution methods (see Newell \& Simon, 1972).

Our view is that a problem solver learns by constructing elements of a table of connections between features of the task environment and solution methods. When enough data have been assembled in such a table, the problem solver induces rules that specify which situations are present and which solutions are good in each instance of the situation. The induction of these rules based upon the data in the table of connection could be accomplished by an induction process that maps classes of stimulus values into utilities for solution methods. Huesmann and Cheng (1973, p. 126) state this more formally as follows: "To induce a rule for solving a problem one must find a function $f\left(X_{1}, X_{2}, \ldots X_{p}\right)$, such that if $X_{1}, X_{2} \ldots X_{p}$ are stimulus variables and $y$ is a variable representing the utility of a particular solution method, then $f\left(X_{1}, X_{2}, \ldots X_{p}\right)=y$ for all observed instances of $\left(X_{1}, X_{2}, \ldots X_{p}, y\right)$.

Less formally, Simon and Lea (1974, pp. 109-110) have expressed this procedure as follows: (1) There is a problem space whose elements are knowledge states. (2) There are one or more generative processes (operators) that take a knowledge state as input and produce a new knowledge state as output. (3) There are one or more test processes for comparing a knowledge state with specification of the problem state and for comparing pairs of knowledge states and producing differences between them. (4) There are processes for selecting which of these generators and tests to employ, on the basis of information contained in the knowledge states.

And still less formally, we can state that, given that the machine or human problem solver has already encountered a number of features in the problem space, he or she can determine the direction in which to continue to search by two kinds of decisions: (1) selection of a knowledge state from among those already encountered, and (2) selection of an inference rule to apply at the node in order to reach a new knowledge state.

Translating this into a rule-induction system that learns a hypothetical mental health decision environment such as one represented earlier, we refer the reader to the inductive discovery system developed by Langley $(1979$, Note 1) that asks the user (computer program or human) a number of questions about the task the system is to solve and about the solution. Our system, which works somewhat like Langley's, uses the following methods to teach the human the elements of the mental health decision problem:

(1) Gathering symptom, disease, and other similar data: A set of productions (see Newell \& Simon, 1972, pp. 32-34) or computer subroutines asks the user (com- 
puter display) a number of questions about the task the system is to solve. As the data are gathered, the system constructs a set of more specific productions to carry out the experiment. Simultaneously with the gathering of the problem features, then, the system collects connections data for the problem solutions.

(2) Detecting regularities: A second set of productions are responsible for discovering regularities in the data collected by the first set. The system's regularity detectors can be divided into a set of constancy detectors and a set of trend detectors. These can deal with nominal and numerical data, respectively, and lead to the postulation of generalizations about the connections between the problem features and solutions.

(3) Computing higher level values: Another set of productions is used to compute the values of new attributes that the system presents. Included in this set are productions that discover and compute independent and dependent values that may, in turn, generalize to still higher level values.

(4) Testing hypotheses: A final set of productions were developed that are responsible for testing generalizations that emerge from the prior subroutines. When a generalization is first made, a set of preexisting test productions will be set in motion to compare the known data with the generalization. When the problem space is deterministic, the test productions will require a perfect comparison. In an uncertain, probabilistic task, the generalization can be less perfect.

The next step following devising a system for inductive rule learning is to display the problem task features to humans on a cathode-ray tube. The environmental features can be displayed in manageable chunks, and the problem solver is to interact with the system and can ask for more information about other features of the environment.

The initial givens to the subject and the complexity of the problems to be solved are raised along several dimensions of complexity. For example, the initial number of symptoms, diseases, differential tests, and treatments can be made more or less complex, depending on what probabilities are assigned to their covariations, and likewise, the seriousness of the consequences of misdiagnosis can be adjusted according to prespecified values. Approximately 10 problems can be presented to each of five subjects.

While the problem solving is underway, verbal protocols are obtained, as well as the solution times of the participating subjects. The idea of obtaining "thinkingaloud" protocols as well as other records of subjects' modes of proceeding (i.e., pointing at various displayed task features with a light pen) is to construct models about human problem solving. These models are in the form of simulation programs that produce two types of output that can be compared with the solutions humans generate: The program prints a protocol of its solution process and it computes the simulated time needed to reach any point in the solution process. To test whether these models predict human performance, humans and computers will be compared in their learning of problem spaces and solutions.

\section{DISCUSSION AND CONCLUSION}

What do the preceding considerations tell us about human performance and decision making in mental health settings? The main insight to be gained from our use of the computer in the foregoing novel ways is the knowledge that computers can be used as "windtunnels," as it were, for simulating a variety of mental health and other clinical environments. Secondarily, we gain knowledge about apparent tradeoffs between computational and cognitive efforts in reaching certain decisions and about the adaptability of both computers and humans to new problem environments. The extent to which our approach provides useful information for specific environments, of course, depends on how well our problem tasks share features with real-world environments.

Our approach should also provide insights about "optimality" in non-mental health decision making spheres. There is a growing realization among decision scientists (see Einhorn, D. Kleinmuntz, \& B. Kleinmuntz, 1979) that real-world decision problems should not be considered exclusively in terms of mathematical optimality. It may be more interesting to give a good description of the problem and its solution than to compute what constitutes rigorous or correct solutions.

Clearly, the potential for elaboration of types of strategies and extrapolation to other decision tasks is immense. The general approach of devising artificial environments that simulate real-world tasks could be a powerful one, and it should be useful in generating interesting hypotheses about human judgment. As these are developed and investigated, perhaps we can give some insights into the cognitive processes that underlie decision making in problem spaces that resemble a variety of demanding decision environments.

Finally, our research should lend itself to restructuring existing real-world environments such as DSM III. Thus, while we dealt mainly with hypothetical symptoms, signs, disorders, treatments, and so on, our system can be used quite readily to discover what taxonomic features of the current diagnostic nomenclature should be modified or elaborated in order to create a more scientifically sound nomenclature and one that will facilitate better decision making.

\section{REFERENCE NOTE}

1. Langley, P. Bacon 1: A general discovery system (CIP Working Paper No. 383). Pittsburgh, Penn: Department of Psychology, Carnegie-Mellon University, 1978. 


\section{REFERENCES}

American Pgychintric Association. Diagnostic and statistical manual of mental disorders (DSM III). Washington, D.C: Author, 1980.

Brooks, R., \& KLEInmuntz, B. Design of an intelligent computer psychodiagnostician. Behavioral Science, 1974, 19, 16-20.

Einhorn, H. J., Kleinmuntz, D. N., \& Kleinmuntz, B. Linear regression and process-tracing models of judgment. Psychological Review, 1979, 86, 465-485.

Huesmann, L. R., \& Cheng, C. A theory for the induction of mathematical functions. Psychological Review, 1973, 80, 126-138.

Kleinmuntz, B. Personality test interpretation by digital computer. Science, 1963, 139, 416-418.

KLeinmuntz, B. Personality test interpretation by computer and clinician. In J. Butcher (Ed.), MMPI research developments ana clinical applications. New York: McGraw-Hill, 1969.

Kuenmuntz, B. The computer as clinician. American Psychologist, 1975, 30, 379-387.

KleinmunTz, B., \& McLean, R. S. Diagnostic interviewing by digital computer. Behavioral Science, 1968, 13, 75-80.

Kleinmuntz, D. N., \& KLeinmuntz, B. Models of judgment in hypothetical environments. Behavioral Science, 1981, 26, 294-305.

Lanoley, P. Rediscovering physics with Bacon 3. Proceedings of the 6th IJCAI-Tokyo, 1979, 1, 505-507.

Newell, A., \& Simon, H. A. Human problem solving. Englewood Cliffs, N.J: Prentice-Hall, 1972.

Simon, H. A., \& LeA, G. Problem solving and rule induction: A unified view. In L. W. Gregg (Ed.), Knowledge and cognition. Potomac, Md: Erlbaum, 1974. 